\title{
Anthony C. Homan* \\ Role of BCA in TIGER grant reviews: common errors and influence on the selection process
}

\begin{abstract}
As directed by the American Recovery and Reinvestment Act of 2009, the US Department of Transportation (DOT) created the Transportation Investment Generating Economic Recovery (TIGER) discretionary grant program for surface transportation infrastructure projects. Through 2013, there have been five rounds of the grant program. TIGER uses a multi-step competitive application process to award surface transportation funds. TIGER applications are initially screened by US DOT's staff of technical experts. For projects forwarded by the review team, US DOT economic experts then review the applicant's benefit-cost analysis (BCA) and attempt to determine the likelihood that the benefits exceeded costs (i.e. not the applicant's self-determination). The final awardees are then selected by a Review Team of Modal Administrators and DOT Office of the Secretary level officials. The purpose of this paper is to discuss many of the common errors in preparing, and issues in reviewing the applicant's BCA and in making a net benefit determination. A secondary purpose is to determine if the most deserving projects, based on an applicant's BCA and the likelihood that benefits exceeded costs, are more likely to receive grant funding. We do so for the second through the fifth rounds of the program.
\end{abstract}

Keywords: benefit-cost analysis; ARRA; TIGER grants.

DOI 10.1515/jbca-2013-0018

Previously published online July 4, 2014

\section{Introduction}

The American Recovery and Reinvestment Act of 2009 (Recovery Act) was a major stimulus package designed to jump-start the US economy. In particular, the Recovery Act designated over $\$ 48$ billion to the US Department of Transportation (DOT) to create jobs and improve infrastructure. Of the $\$ 48$ billion, $\$ 1.5$ billion was directed

*Corresponding author: Anthony C. Homan, Department of Transportation, 1200 New Jersey Ave., SE Washington District of Columbia 20590, USA, Phone: +202-366-5406, e-mail: anthony.homan@dot.gov 
to a new discretionary grant program. This resulted in the creation of the Transportation Investment Generating Economic Recovery (TIGER) grant program.

In May of 2009, US DOT published the TIGER Notice of Funding Availability (NOFA, 2009) for the first competition of TIGER in the Federal Register. DOT conducted additional rounds (II-V) of TIGER in 2010, 2011, 2012, and 2013. TIGER is a competitive grant program that is open to states, municipalities, and other governmental bodies. TIGER provides funding for transportation infrastructure projects including highways, bridges, transit, freight and passenger rail, and port infrastructure. As a funding structure, TIGER is a departure from the US DOT's normal formula-based infrastructure funding. Instead, TIGER's competitive selection process relies on a merit-based process to allocate infrastructure funds. The US Government Accountability Office (GAO) has urged the US DOT to develop funding opportunities that use performance metrics and to allocate funds to regional and national priorities as opposed to formula funding (GAO, 2011b). The TIGER grant program marks a significant step toward implementing the type of program the GAO is promoting.

TIGER uses an application process to differentiate projects and determine which projects receive funding. For the first round of TIGER, all applicants seeking grants of more than $\$ 20$ million but $<\$ 100$ million were required to provide an estimation of the expected benefits of the project. All applicants seeking more than $\$ 100$ million were required to provide a well-developed benefit-cost analysis (BCA) (NOFA, 2009). For subsequent rounds of TIGER, essentially all applicants had to provide a BCA. US DOT staff rated the accuracy and reliability of the BCA of each application that made it past an initial evaluation. Staff also determined the likelihood that the benefits exceeded costs (i.e. not the applicant's selfdetermination). Both were some of the several factors that senior DOT politically appointed leadership were to consider in awarding TIGER grants. If the quality of the $\mathrm{BCA}$ and higher net social benefits are important factors in determining an awarding a TIGER grant, then we would expect to see a revealed preference for those characteristics.

However, Homan, Adams, and Marach (2014) found that both the rating of the BCA and the likely net benefits were generally not significant factors in the final selection of projects for the first round of TIGER. They also found that the general level quality of the submitted BCA's was not good and that there was a high level of uncertainty in determining likely net benefits. One would expect the greater the uncertainty surrounding both the quality of the analysis and the likelihood of net benefits, the less likely that these two factors would influence the selection process.

Following TIGER I, the US DOT took additional steps to try to improve the quality of the applicant's BCAs. This included conducting webinars and providing 
guidance in the NOFAs on conducting BCA's and what constitutes societal benefits. (NOFA, 2010, 2011, 2012, 2013). Much of the initial guidance focused on correcting deficiencies found in benefit estimation in TIGER I, with modifications following subsequent rounds. In doing so, the expectation was to both improve the quality of the analysis and the influence of BCA in selecting awards.

The organization of the paper is as follows. We first describe the TIGER program review and selection process. We then describe some of the issues encountered in reviewing the BCA's and types of mistakes frequently made in applicant's calculation of benefits. Then we describe how DOT coded and presented the results to DOT leadership. Finally, we discuss the extent that both the quality of the BCA and the likelihood of net benefits influenced the selection process for TIGER rounds II-V and whether the quality increased over time.

\section{Review process}

The US DOT created a multi-step application process to ensure that applicants were assessed on everything from the accuracy of the BCA to the readiness of the project. All applications were first reviewed based on the designated primary and secondary selection criteria. Primary criteria related to how well the applications contributed to the long-term strategic outcomes for the TIGER program. These outcomes are: (1) State Of Good Repair; (2) Economic Competitiveness; (3) Livability; (4) Sustainability; and (5) Safety. State of Good Repair refers to improving the condition of existing transportation facilities and systems. In particular, it deals with minimizing life-cycle costs and in improving resiliency. Economic Competitiveness deals with improving the long-term efficiency of using the transportation network in the movement of workers or goods. In particular, it deals with reducing the costs of transporting cargoes and increasing productivity. Livability deals with increasing transportation choices and access to transportation services for people and communities across the country. Sustainability refers to improving energy efficiency and reducing greenhouse gas emissions. Safety refers to improving the safety of US transportation facilities and systems. Primary criteria also included the extent to which a project created jobs and provided economic stimulus.

Secondary criteria listed in the NOFA were: (1) Innovation; and (2) Partnership. Innovation refers to use of new methods, including financing, to address TIGER's long-term strategic outcomes. Partnership refers to the extent to which the applicant partners with other stakeholders and across multiple jurisdictions as well as the extent to which the applicant leverages funds from private and other public sources. The NOFAs provide more detail on both primary and secondary criteria. 
The review process primarily involved five types of teams. These teams were: (1) Evaluation Teams; (2) a Project Readiness Team; (3) an Economic Analysis (Econ) Team; (4) a Control and Calibration (CC) Team; and (5) a Review Team (RT). The Evaluation Teams were comprised of career staff experts from across the modal administrations who reviewed the proposals for technical feasibility and likelihood for contributing to the strategic outcomes and other aforementioned primary and secondary criteria. The Evaluation Team then rated the projects as highly-recommended, recommended, or not recommended based on the projects contribution to the strategic outcomes of TIGER. TIGER also included a review of the environmental status and other project readiness factors to ensure that projects were shovel-ready.

The CC Team ensured consistency in the ratings process across the Evaluation Teams and the quality and integrity of the overall process. During TIGER I, the CC team separately forwarded a substantial share of projects to the RT, and in particular projects that received a grant. Indeed, Homan et al. (2014) found that single most important factor in receiving an award was whether the CC Team had forwarded a project to the RT. This CC Team relied on other considerations besides the published primary and secondary evaluation criteria used by the Evaluation Team (e.g. geographical distribution) in forwarding projects to the RT. However, examining the influence of the CC team over and above the Econ Team's review of the analysis (see below) is outside the scope of this paper.

The Econ Team reviewed all of the highly-recommended projects forwarded by the Evaluation Teams and the projects forwarded by the CC Team for adequacy of the analysis of costs and benefits. Consequently, the Econ team only reviewed a subset of the total applications. The Econ Team also provided an assessment of the likely benefits and whether those benefits would likely outweigh the costs of the project.

The RT had the responsibility of selecting a suite of projects that would equitably distribute funds across the nation, across rural and urban areas, contribute to the achieving TIGER's long-term strategic outcomes, and satisfy the other primary and secondary criteria (GAO, 2011a). The RT consisted of the DOT Modal Administrators, the Deputy Secretary of DOT, the Under Secretary and Assistant Secretary for Policy, and other higher ranking DOT officials (e.g. Deputy Assistant Secretaries).

To assist the RT in their deliberations, the leaders of the Evaluation Teams and the Econ Team conducted formal briefings on all projects forwarded to the RT. During these briefings, the Econ Team discussed both the quality of the analysis and their assessment on the projects likely net benefits. There was a great amount of uncertainty surrounding many of these assessments due to errors in how many of the applicants prepared the BCAs. The next section discusses many of the more common errors. 


\section{Common types of BCA errors}

The Econ Team encountered several issues with the quality of the BCA's during the reviews. These issues in turn affected the ability to precisely quantify benefits. These issues included identifying the correct baseline, analyzing project alternatives, correctly identifying the affected population, transfers, speculative benefit estimation, and lack of transparency.

\subsection{Baselines}

Applicants often had a difficulty establishing the correct baseline and how a project would change that baseline. Consequently, they would not be estimating benefits on the actual proposed TIGER funded project. In turn, this made it difficult for DOT to correctly gauge the benefits.

Ideally, applicants should measure costs and benefits of a proposed project against a baseline (also called a "base case" or a "no build" case). The baseline should be an assessment of the way the world would look if the project did not receive the requested TIGER Discretionary Grant funding. Sometimes, it is reasonable to forecast that that baseline world resembles the present state. This is especially so for the beginning of the analysis. A common error was using conditions projected for a distant future date as a baseline throughout the analysis. For example, using projected traffic levels for 2030 as the baseline traffic to generate benefits for all the earlier years is incorrect as many of the earlier years would have had little or any congestion. Using the incorrect baseline for all the earlier years inflates the estimated benefits. Conversely, applicants using the correct starting baseline need to adjust future years for projected traffic levels in those years. Not doing so would underestimate future travel time savings. However, we did not find this to be a common error. Applicants should match forecasts of usage levels to the corresponding year to correctly estimate benefits. It is important to factor in any other projected changes (e.g. completion of already planned and funded projects) that would occur even in the absence of the requested project. For example, estimates of the congestion-relief time savings benefit from a proposed road project can be inflated if the analysis does not consider the positive effects of other nearby road projects. A common error in many BCAs was that the analyses ignored the effect of other planned infrastructure projects. This created a positive bias towards inflating benefits.

It is also important that the applicant assume the continuation of reasonable and sound management practices in establishing a baseline. Assuming, for example, a baseline scenario in which the owner of the facility does no 
maintenance on the facility and ignores traffic problems and maintenance is not realistic and will lead to the overstatement of project benefits.

Baselines also need to be realistic in the transportation assumptions that they make. However, many BCAs relied on unrealistic assumptions that exaggerated benefits. For example, if a project would construct a short freight rail spur from a railroad mainline to a particular facility, it is unrealistic to assume that, in the absence of the project, individuals would ship cargo by truck for thousands of miles, whereas they would ship the same cargo by rail with the project. A realistic description of current traffic would more likely have current cargo traffic going by rail for most of the distance, and then by truck for the relatively short distance over which rail transportation is not available.

There are cases where a grant may accelerate completion of the project that an applicant already was going to build. The benefits and costs in this case should thus be limited to the marginal benefits (and marginal costs) of completing the project in a shorter period of time and including the cost of expending resources on the project sooner than otherwise planned (i.e. a "now versus later" comparison). However, a common BCA error with these types of projects was to assume all project benefits came as a result of the TIGER grant.

Frequently, BCAs did not demonstrate that the proposed project has independent utility. Sub-components of a larger project may have little or no transportation value in the absence of the other components. For example, a ramp to an undeveloped site does not have much utility if the site does not get developed. While the project may increase the likelihood of development of the site, the application must be realistic in assessing the probability that transportation improvements will actually induce development.

Another issue was in applicants grouping together several unrelated projects where each of the projects had independent utility. In some cases, many of the projects had negative net benefits (either as described by the applicant or more frequently after Econ Team review) and only by grouping them with a larger positive net benefit project could the applicant justify the combined group of projects. In other cases, after Econ Team review, the combined negative effect of the negative net benefit projects outweighed the positive effect of the larger project.

Many BCAs also failed to make clear exactly what portions of the project form the basis of the estimates of benefits and costs. It is incorrect to claim benefits for the entire project but only count as costs the costs of the portion of the project funded by the TIGER Discretionary Grant. Thus, it would be incorrect to attribute all the benefits from a new port facility to a TIGER Discretionary Grant when the costs that are counted only cover the portion of the project funded by the TIGER Discretionary Grant, for example, paving a loading area. Similarly, many BCAs for road projects did not estimate travel time savings solely from the project funded 
by the requested grant, but rather from other related projects not funded by the requested grant. When this occurred, it was usually not possible to isolate the travel time savings solely from the project requested by the grant. In all cases where there was a mismatch between the basis for calculating benefits (e.g. larger project) and the basis for calculating costs (e.g. TIGER funded portion), there are issues with the quality of the analysis. However, there may also be issues with the incentives to do more careful analysis and the historical desire to show high net benefit numbers (see Transparent and Reproducible Analysis).

\subsection{Alternatives}

BCAs often did not present and consider reasonable alternatives in the analysis. Applicants should evaluate smaller-scale and more focused projects for comparison purposes. For example, if an applicant is requesting funds to replace a pier, it should also analyze the alternative of rehabilitating the current pier. For example, an applicant can compare the maintenance costs that would be required after rehabilitating an existing pier with those that would be required after building a new one. Similarly, if an applicant seeks funds to establish a relatively large streetcar project, it should also evaluate a more focused project serving only the more densely populated corridors of an area. Some lines in a project make more economic sense than others. We often found that after Econ Team review, many lines (or sub-components of a line) had negative benefits. In grouping the whole project together, the lines with negative benefit could dilute the total benefits and could end up resulting in a project the Econ Team found to be not net beneficial. In cases like these, an application focused on the best alternative would yield higher net benefits after Econ Team review.

\subsection{Affected population}

Many BCAs were not able to correctly identify the population affected by the proposed TIGER investment. In many ways, this was linked to not being able to correctly identify the baseline. It is important that applicants match the types of impacts to the corresponding affected population (group and number of affected entities). For example, two primary groups are users of freight rail and operators of private vehicles.

For freight rail improvements (e.g. at a port or via a new bridge), applicants often claimed benefits from reduced shipping costs and reduced emissions from diverting traffic from truck to rail. Unfortunately, there was frequently not any 
basis in the claimed diversion levels. Similarly, forecasts for increased freight traffic were often unreasonably high and without basis. A common error was matching freight levels in future years to the first few years in the project; this resulted in inflated cost savings. The inability to provide a realistic justification for the affected population (e.g. baseline and forecast traffic) was a frequent issue with these BCAs.

Correctly identifying the affected population was a particular issue when estimating benefits from travel time savings. These benefits can result from transportation improvements whose purpose is to expand capacity or improve state of good repair. Where this is the case, BCAs need to clearly demonstrate how the travel time savings are experienced by the number of affected drivers and passengers. If travel time savings vary over time, the BCA must clearly show savings by year. Frequently, BCAs did not correctly link the reduction in travel time correctly. The most common error was matching savings in future years to the first few years in the project (see baseline).

\subsection{Transfers}

Transfers are not benefits. Perhaps the most common error in BCAs was counting transfers as benefits. BCAs should distinguish between real benefits and transfer payments. Benefits reflect reductions in real resource usage and overall benefits to society, while transfers represent payments by one group to another and do not represent a net increase in societal benefits. In the case of job creation, for example, every job represents both a cost to the employer (paying a wage) and a benefit to the employee (receiving a wage), so it is a transfer payment, rather than a net benefit. However, since the initial transfer is from federal taxpayers to local government agencies, the net transfer is from federal taxpayers to workers. ${ }^{1}$

While wages are a transfer payment, increases in the productivity of the labor force, measured by increases in how much workers produce per hour, can

\footnotetext{
1 In the existence of unemployed or underemployed labor, job creation can be real benefit since excluded labor services exceed the opportunity cost of unemployed workers' (involuntary) leisure time. However, there is uncertainty as to the percentage of workers drawn from the ranks of those already employed in the construction trades, in particular the more specialized labor trades (i.e. that require training) as opposed to the percentage of workers drawn from the ranks of the unemployed. There is also uncertainty as to the extent that all unemployed labor can quickly shift into some of the more specialized labor trades engaged in construction of infrastructure projects. For these reasons, for the purposes of the BCA, DOT assumed a full employment labor market. This also facilitated comparison across projects with different unemployment rates in different regions.
} 
be included as a benefit of the project, but these benefits must be carefully measured and justified to be included. With respect to economic development, providing estimates of capital investments or property tax revenues are not legitimate benefits in a benefit-cost analysis. For example, while the tax is a benefit to the tax assessor it is a cost to the taxpayer. These transfers are commonly included in "economic impact analyses;" an economic impact analysis is not acceptable as a substitute for a benefit-cost analysis. However, many BCAs (especially for projects focused on economic competitiveness) relied primarily on economic impact analysis to estimate benefits.

Another example of a transfer that BCAs listed as benefits was a transfer of business from one location to another. For example, this included port/rail projects whose purpose is to take away business from competitors. In these cases, the BCAs would typically include the volume of traffic shifted as a benefit. However, the transportation cost savings (if any) and the like from shifting traffic to a more convenient location would be the benefit. Many BCAs also included employment or output multipliers that purport to measure secondary effects as societal benefits. However, these secondary effects represent either double-counting of direct benefits or transfers that occur as the primary benefits ripple through the economy. In either case, they do not represent additional benefits.

\subsection{Property value increases}

Many BCAs for transit projects showed most of the benefits resulting from property value increases. However, many were substantially inflated or poorly justified. BCAs should carefully net out other effects before taking benefits from property value increases (e.g. from a transit station). Applicants must net out any property value increases that result from time savings or other benefits that have already been counted, and the only other reason that any excess can arise is the existence of agglomeration, environmental or other benefits that are external to individual developers making investments on the site served by new or improved transit service. The analysis should also consider to what extent an increase in land values induced by the project in one area causes a reduction in land values in some other area. Applicants can only count the net increase in land value as a benefit. However, many BCAs claiming these benefits failed to do any of the above in any meaningful way. In some cases, BCAs simply asserted that there was a property value increase net of time savings. In other cases, BCAs used benefit transfer methods as opposed to survey methods to estimate the value of the expected property value increase from transit or other transportation improvements. When using benefit transfer methods, BCAs need to take great care to 
satisfy the selection criteria and the disqualifying criteria noted in OMB Circular A-4 (2003, p. 25). Meeting all these criteria is difficult, but an applicant should satisfy most of them before applying this approach. Most BCAs using the benefit transfer approach failed to do so.

Another error was in treating property value benefits as an annuity (especially in earlier rounds). Any claimed societal benefit from a property value increase is only a one-time "stock" benefit and not a stream of benefits accruing annually. The former approach reflects poorly on the quality of the analysis but it also may reflect historical incentives to report high net benefit numbers. It may also reflect the expectation that no one would thoroughly review the societal benefits estimates (see Transparent and Reproducible Analysis).

\subsection{WTP for bicycle benefits}

BCAs trying to estimate benefits from bike trails often relied on a non-peer reviewed stated preference study from Minnesota that relied on 168 respondents willingness to travel (WTT) a number fixed additional minutes to use a trail instead of riding on the road. The majority of the respondents were not regular cycling commuters. The BCAs would take the estimated number of minutes (about $20 \mathrm{~min}$ ) to move from cycling on the road to a trail, monetize it using the value of travel time, and then apply it to a population of all existing road and forecasted trail users. At $20 \mathrm{~min}$, daily societal benefits would be over $\$ 8$ for each bicycle commuter (round-trip) using the trail. The approach is a proxy for changes in consumer surplus. For new riders (the triangular component of the consumer surplus trapezoid) the approach results in benefits always being twice as high for any given WTT. For existing riders (the rectangular component of the consumer surplus trapezoid) the approach yields consistent results. However, to be consistent the actual cost of the commute (in minutes spent) would need to remain constant. Most projects proposed trails that would have resulted in substantially increased travel times for existing commuters, thus eroding the rectangular component of the consumer surplus trapezoid. This also produced the counter-intuitive results of increased travel times coupled with substantial benefits.

The value of benefits is also very sensitive to the WTT to use a trail. A fixed, one-size-fits-all estimate for willingness to travel to use a trail is problematic because of the different trip times (often fairly short) for many bicycle commuters. Many existing commuters would have rides of less duration than $20 \mathrm{~min}$ so it is not likely you'd drastically increase total commuting time to use a trail. Actual WTT vary and in many cases would be small. This is especially so for projects with limited scope (e.g. short trail length). For example, if WTT were only 5 min 
the benefits would be substantially smaller. There is also the issue of the applicability (geography, demographics, etc.) of the aforementioned study for benefit transfer purposes to other locations. This, and the uncertainty surrounding the study's validity, are not trivial matters given the large consumer surplus benefits that the approach generates.

What these BCAs did not generally do was analyze any benefits from modeshift (private vehicles to bicycles). We generally did not see attempts to estimate benefits from reduced congestion for remaining drivers, time savings for modeshifters (if applicable), savings from reduced vehicle operating costs, and Sustainability benefits from reduced vehicle emissions.

\subsection{Transparent and reproducible analysis}

Many BCAs did not make the results of their analyses transparent and reproducible. In many cases, there was not any way to reproduce the results or even follow the calculations driving the estimates. These BCAs did not provide enough information so that a Department reviewer could follow the general logic of the estimates (and, in the case of spreadsheet models, reproduce them). This, of course, generates significant amount of uncertainty concerning the results.

A common error in this area was for the BCA to only provide links to large documents or spreadsheets as sources. Many of these BCAs did not clearly cite all outside data sources with the corresponding page number (or cell number, for a spreadsheet). In particular, many BCAs did not include a thorough verbal description of how they did the calculation (e.g. references to tabs and cells in the spreadsheet). This made it difficult to confirm results, especially given the relatively short time frame that was available to review BCAs. Another error was when BCAs relied on "pre-packaged" economic models to calculate net benefits. In most of these cases, the BCAs did not provide annual benefits and costs by benefit and cost type for the entire analysis period (including forecast year traffic volumes). Additionally, they generally did not provide a detailed explanation of the assumptions used to run the model (e.g. peak traffic hours and traffic volume during peak hours, mix of traffic by cars, buses, and trucks, etc.). Both of these factors made it impossible to reproduce the results or to put any level of certainty on the results of the analysis.

When done correctly, a BCA is an ex-ante tool for decision making (e.g. project selection). However, many of the aforementioned issues arise since BCAs usually are done ex-post of the project selection. As such, there is a limited incentive to do a quality BCA. The historical tendency is to view BCAs as a "box checking” exercise, and at that a box that has to show high net benefits to justify the project. This is 
especially so when the BCA is not linked to project selection. The bias is to show high net benefits since there is a "strategic bias" to overstate benefits and underestimate costs (Flyvbjerg, Holm, and Buhl, 2005). There is also the expectation (especially at the state and local level) that BCAs won't be heavily scrutinized for quality. At the margin, there is less of an incentive to produce quality, transparent work and there is an incentive to inflate societal benefits since the expectation is that no one will carefully check the estimates. Conversely, when someone does review the BCA, it may be from "Guardians" in line agencies that view BCAs as a naïve and impractical tool and instead focus on revenue-expenditure analysis (Boardman, Greenberg, Vining, and Weimer, 2006). At the margin, this creates an incentive is to have the BCA focus more on transfer activities and less on societal benefits.

Because of all these issues, it was not readily possible to precisely quantify benefits for most projects. While in some cases, a reviewer could subtract out incorrectly included benefits (e.g. transfers) to arrive at a benefit-cost ratio (BCR), in most cases they could not. For many of the submitted BCAs, a reviewer would have to independently do the BCA to generate a BCR. This was not clearly possible given staff constraints and the tight review window for each TIGER round. However, it was important to still be able to provide information on both the quality of the analysis and more importantly the likely net benefits of each project to the RT. The next section describes how the Econ team did so.

\section{Data}

To provide the RT with meaningful information on the BCAs, the Econ team needed to develop metrics to describe both the analysis and the likelihood that the project had net benefits. The metrics also allow us to conduct statistical analysis on whether the quality of the BCA and the likelihood that benefits exceeded costs (net social benefits) influenced whether or not DOT selected a project for funding.

\subsection{Quality of BCA}

The Econ Team was responsible for evaluating the BCA portion of the application for accuracy. They did so by assigning qualitative categorical ratings of very useful, useful, marginally useful, or not useful. Table 1 summarizes the rating criteria. We next quantify these ratings by creating a BCA-Usefulness variable. We do so by assigning an ordinal scale to the ordered categorical ratings. On the 
Table 1 Econ team rating criteria.

\begin{tabular}{ll}
\hline Rating & Description \\
\hline Very useful & The economic analysis (i) is comprehensive (quantifying and monetizing \\
the full range of costs and benefits for which such measures are reasonably \\
available), (ii) attempts to capture the dynamic effects of transportation \\
investments on land use, as well as the economic effects of transportation \\
investments on households, (iii) helps the Department organize information \\
about, and evaluate trade-offs between, alternative transportation \\
investments, and (iv) provides a high degree of confidence that the benefits of \\
the project will exceed the project's costs. \\
The economic analysis (i) identifies, quantifies, monetizes, and compares \\
the project's expected benefits and costs, but has minor gaps in coverage of \\
benefits and costs, or fails in some cases to quantify or monetize benefits and \\
costs for which such measures are reasonably available, and (ii) provides a \\
sufficient degree of confidence that the benefits of the project will exceed the \\
project's costs. \\
The economic analysis (i) identifies, quantifies, monetizes, and compares the \\
project's expected benefits and costs, but has significant gaps in coverage, \\
quantification, or monetization of benefits and costs, or significant errors in \\
its measurement of benefits or costs, and/or (ii) the Department is uncertain \\
whether the benefits of the project will exceed the project's costs. \\
useful
\end{tabular}

ordinal scale, a very useful analysis was coded as four, useful as three, marginally useful as two, and an analysis that was not useful was coded as one. We apply the scale to the categorical ratings that DOT's Econ Team assigned to each project.

\subsection{Net social benefits}

The Federal Register NOFAs for TIGER has discussed the importance of projects providing positive net social benefits. Specifically, it noted the DOT will not award a TIGER Grant if "the total benefits of a project are not reasonably likely to outweigh the project's costs." As noted in the previous section, there were several issues in most of the applicant's estimation of benefits. Given the time frame involved in reviewing the application, the Econ Team did not have the available time to separately quantify the benefits of each proposed project. However, it was important to be able to provide information on the likely net benefits to the RT. 
To do so, the Econ Team provided a subjective rating of the likelihood that a project's benefits would outweigh its costs, which was based on the team's assessment of the uncertainty surrounding (or the reliability of) the benefits and cost estimates submitted in support of each project. This was an independent assessment and was different than the self-assessment provided by the applicant. The Econ Team rated projects as follows: benefits greater than costs; uncertain but benefits likely to outweigh costs; uncertain; uncertain but costs likely to outweigh benefits; and benefits less than costs.

As with the BCA, we also quantify these ratings. We do so by assigning an ordinal scale to the ordered categorical ratings. We used the Probability of Net Benefits variable to code the Econ Team's ratings on an ordinal scale of five, four, three, two, and one, respectively; with the range from 5 being "benefits greater than costs" to 1 being "benefits less than costs." Table 2 summarizes the rating scales for both BCA-Usefulness and Probability of Net Benefits.

\section{Methods and results}

If the quality of the BCA and higher net social benefits are important factors in determining an awarding a TIGER grant, then we would expect to see a revealed preference for those characteristics. To determine if there were any such preferences, we made pair-wise comparisons of average BCA-Usefulness and Probability of Net Benefits variables of the awardees to those of the non-awardees to determine if there are any statistically significant differences. The null hypothesis (or default position) is that means are equal for awardees and non-awardees.

H1: average score awardees=average score non-awardees

We used the statistical test of equality between means (average values) to test the hypothesis. We do so for individual TIGER rounds two through five (II-V).

Table 2 Rating scale summary.

\begin{tabular}{lrlr}
\hline BCA-Usefulness & $\begin{array}{r}\text { Rating } \\
\text { scale }\end{array}$ & $\begin{array}{l}\text { Probability of Net } \\
\text { Benefits }\end{array}$ & $\begin{array}{r}\text { Rating } \\
\text { scale }\end{array}$ \\
\hline Very useful & 4 & Benefits>Costs & 5 \\
Useful & 3 & Uncertain/Benefits $>$ Costs & 4 \\
Marginally useful & 2 & Uncertain & 3 \\
Not useful & 1 & Uncertain/Costs $>$ Benefits & 2 \\
& & Costs $>$ Benefits & 1 \\
\hline
\end{tabular}


The basis for the test is a single-factor, between-subject analysis of variance (ANOVA). If awardees and non-awardees have the same mean, then the variability between those groups should be the same as the variability within each group. The test statistic for the ANOVA test is an F statistic. Equation (1) below shows the equation for the test.

$$
F=\frac{S S_{b} /(G-1)}{S S_{w} /(N-G)}
$$

In the equation, $N$ is the total number of observations and $G$ is the number of groups. $S S_{b}$ is the sum of squares between subgroups and $S S_{w}$ is the sum of squares within subgroups. Equations (2) and (3) show the calculation of $S S_{b}$ and $S S_{w}$, respectively. In the equations, $X_{g}$ is the sample mean within group $g, X$ is the overall sample mean, $x_{i g}$ is an individual observation within a group, and $n_{g}$ is the number of observations in the subgroup (Quantitative Micro Software, 2007).

$$
\begin{aligned}
& S S_{b}=\Sigma^{G} n_{g}\left(X_{g}-X\right)^{2} \\
& S S_{w}=\Sigma^{G} \Sigma^{n g}\left(x_{i g}-X_{g}\right)^{2}
\end{aligned}
$$

The larger the F-test statistic (the lower the probability) the more likely there is a significant difference between awardees and non-awardees. Generally speaking, probability levels of 0.1 or less ( $90 \%$ confidence) are statistically significant. This implies that the likelihood of the difference happening by chance is $10 \%$ or less. The lower the probability of an event occurring by chance the more likely the occurrence is due to a preference for a causal factor. In this case, the causal factors would be if BCA-Usefulness and Probability of Net Benefits played a role in the award process.

Table 3 shows the results for the pair-wise comparisons of average BCAUsefulness and Probability of Net Benefits variables of the awardees to those of the non-awardees for the combined rounds, and the individual rounds of TIGER II-V. For the combined rounds, both BCA-Usefulness and Probability of Net Benefits were significantly higher for awardees. However, the results for earlier rounds (especially TIGER II) are driving the results. The Probability of Net Benefits variable was higher for awardees in rounds II-III and the differences were statistically significant. While the variable was also higher in rounds IV-V, the differences were not significant. For round $\mathrm{V}$, the difference does not quite rise to an accepted level of significance $(\mathrm{p}=0.19)$. The result for the BCA-Usefulness variable is less robust. While the variable was higher in both rounds II-III and V, only the difference in round II is significant. TIGER II appears to be the round where both 
Table 3 Tests of equality for BCA-Usefulness and Probability of Net Benefits variables (awardees \& non-awardees).

\begin{tabular}{lrrrr}
\hline Variable & $\begin{array}{r}\text { Average } \\
\text { awardees }\end{array}$ & $\begin{array}{r}\text { Average non- } \\
\text { awardees }\end{array}$ & F-value & Probability \\
\hline TIGER II-V & & & & \\
$\quad$ BCA-Usefulness & 2.320 & 2.171 & 2.53 & $0.06^{*}$ \\
$\quad$ Probability of Net Benefits & 3.691 & 3.439 & 5.70 & $0.00^{* * *}$ \\
TIGER II & & & & $0.009^{* * *}$ \\
$\quad$ BCA-Usefulness & 2.333 & 1.908 & 7.34 & $0.02^{* *}$ \\
$\quad$ Probability of Net Benefits & 3.714 & 3.333 & 5.94 & 0.27 \\
TIGER III & & & & $0.06^{*}$ \\
$\quad$ BCA-Usefulness & 2.324 & 2.147 & 1.23 & 0.96 \\
$\quad$ Probability of Net Benefits & 3.568 & 3.274 & 3.65 & 0.37 \\
TIGER IV & & & & 0.00 \\
$\quad$ BCA-Usefulness & 2.277 & 2.284 & 0.00 & 0.19 \\
$\quad$ Probability of Net Benefits & 3.723 & 3.569 & 0.81 & \\
TIGER V & & & & \\
$\quad$ BCA-Usefulness & 2.346 & 2.263 & 0.32 & \\
$\quad$ Probability of Net Benefits & 3.731 & 3.517 & 1.74 & \\
\hline
\end{tabular}

*Significant at a $90 \%$ level of confidence.

** Significant at a $95 \%$ level of confidence.

$\star \star \star$ Significant at a $99 \%$ level of confidence.

${ }^{1}$ For TIGER round III, we exclude 19 applications that had inconsistencies in the reported data for these two variables.

variables influenced the selection process the most. These results would seem to indicate that to the influence of both Probability of Net Benefits and BCA-Usefulness in the selection process, while still somewhat important in round III, has decreased since TIGER II.

We also wanted to test for equality among the different ordinal ratings for both the Probability of Net Benefits and BCA-Usefulness scores [see Equation (1)]. Instead of comparing the average scores of awardees and non-awardees, this is a comparison of the probability of getting an award by each rating category (i.e. the percentage of projects with that rating that received an award). The null hypothesis is that all the rating categories have the same award percentage.

$\mathrm{H} 2$ : Award percentage is equal for all rating scores

If the award percentage is progressively higher (lower) with each increase (decrease) in the rating category, we'd expect that it is likelier for the differences to be significant. Conversely, if the range between the categories is relatively flat 
and the individual award percentages are all close to the total award percentage, then it is less likely that the differences would be significant. However, even if most individual award percentages are close to the total award percentage we can still reject the null hypothesis if there is a significant outlier (e.g. one rating has a much higher award percentage than the average).

We also conduct a simple hypothesis test between the award percentage for each score and the total award percentage for the round. The null hypothesis is the award percentage for the individual rating score is equal to the award percentage for the entire round (or combined rounds).

H3: award percentage for rating score=total award percentage for round

The test statistic for the test is a $Z$ statistic. Equation (4) shows the equation for the test.

$$
Z=\frac{X-X_{r}}{\sigma / N^{.5}}
$$

In the equations, $X_{r}$ is the award percentage for the rating score, $X$ is the overall award percentage for the round, $\sigma$ is the standard deviation for the total award percentage for the round, $N$ is the number of observations in the round. If the award percentage is progressively higher with each increase in the rating category we'd expect to see lower rated projects with a statistically significant lower than average award percentage. We'd also expect to see higher rated projects with a statistically significant higher than average award percentage. If this does not occur, it indirectly indicates that there is an indifference to relative increases in BCA and project quality.

Table 4 shows the results for the Probability of Net Benefits and Table 5 shows the results for BCA-Usefulness. The F-test results for the tests of equality are on the last row. If the results of the Z-tests for the hypothesis tests are significant, we note so for the individual score and round in question. For the combined rounds of TIGER, we reject the null hypothesis of equality for both Probability of Net Benefits and BCA-Usefulness. However, in both cases the results are primarily driven by the high percentage of awarded projects in the top rating category and not necessarily because of progressively higher award percentages by category.

Nearly $45 \%$ of the 94 applications where the Econ team thought the benefits clearly outweighed the costs (Probability of Net Benefits score 5) received an award. Conversely, at the lower end of the scale, none of the applications where the costs clearly outweighed the benefits received an award. However, the award percentages for rating scores 2-4 were fairly similar ranging from and about 25\% with the second lowest score of 2 (uncertain but costs likely to outweigh benefits) 
Table 4 Tests of equality between Probability of Net Benefits ordinal rating scores.

\begin{tabular}{|c|c|c|c|c|c|}
\hline \multirow{2}{*}{$\begin{array}{l}\text { Probability of } \\
\text { Net Benefits }\end{array}$} & \multicolumn{5}{|c|}{ Total count of BC score for round $/ \%$ of count awarded } \\
\hline & TIGER II-V & TIGER II & TIGER III & TIGER IV & TIGER V \\
\hline 1 & $6 / 0^{\star \star *}$ & 0 & $1 / 0^{* * *}$ & $2 / 0^{* * *}$ & $3 / 0^{* * *}$ \\
\hline 2 & $65 / 24.6^{\star * *}$ & $11 / 36.4$ & $15 / 20.0^{\star *}$ & $20 / 20.0^{\star *}$ & $19 / 26.3$ \\
\hline 3 & $234 / 27.8$ & $66 / 25.8^{*}$ & $61 / 27.9$ & $51 / 25.4$ & $56 / 32.1$ \\
\hline 4 & $194 / 28.9$ & $32 / 25.0^{*}$ & $46 / 21.7$ & $56 / 39.3^{\star \star \star}$ & $60 / 25.0$ \\
\hline 5 & $94 / 44.7^{\star \star \star}$ & $19 / 68.4^{\star \star \star}$ & $9 / 77.8^{\star \star \star}$ & $34 / 23.5$ & $32 / 43.8^{\star \star \star}$ \\
\hline ALL & $593 / 30.0$ & $128 / 32.8$ & $132 / 28.0$ & $163 / 28.8$ & $170 / 30.6$ \\
\hline $\begin{array}{l}\text { F-Test } \\
\text { (Probability) }\end{array}$ & $3.53(0.007)^{\star * \star}$ & $4.82(0.00)^{\star \star \star \star}$ & $3.41(0.01)^{\star *}$ & $1.33(0.26)$ & $1.26(0.29)$ \\
\hline
\end{tabular}

* Significant at a $90 \%$ level of confidence.

${ }^{* \star}$ Significant at a $95 \%$ level of confidence.

$\star \star \star$ Significant at a $99 \%$ level of confidence.

to $29 \%$ for the second highest score of 4 (uncertain but benefits likely to outweigh costs) and all were below the total award percentage of $30 \%$. Only the top score of 5 had an award percentage significantly higher than the total award percentage. While the lowest scores $(=1 \& 2)$ were significantly lower than average, the other scores were not significantly different.

For round II-III, the results are similar. For both rounds, we reject the null hypothesis of equality. However, the results are again driven by the high percentage of awarded projects that had a score of 5 . In both cases, that award percentage was significantly higher than total award percentage for the round. In both rounds, the second highest score of 4 had an award percentage below the total

Table 5 Tests of equality between BCA-Usefulness ordinal rating scores.

\begin{tabular}{|c|c|c|c|c|c|}
\hline \multirow{2}{*}{$\begin{array}{l}\text { BCA-Usefulness } \\
\text { score }\end{array}$} & \multicolumn{5}{|c|}{ Total count of BCA-Usefulness score for round/\% of count awarded } \\
\hline & TIGER II-V & TIGER II & TIGER III & TIGER IV & TIGER V \\
\hline 1 & $128 / 29.7$ & $36 / 30.6$ & $26 / 30.8$ & $32 / 31.2$ & $34 / 26.5$ \\
\hline 2 & $253 / 26.9^{*}$ & $56 / 19.6$ & $62 / 24.2$ & $68 / 29.4$ & $67 / 32.8$ \\
\hline 3 & $168 / 29.2$ & $29 / 51.7^{\star * *}$ & $36 / 22.2$ & $48 / 22.9^{*}$ & $55 / 27.3$ \\
\hline 4 & $44 / 52.3^{\star \star \star}$ & $7 / 71.4^{\star \star \star}$ & $8 / 75.0^{\star \star *}$ & $15 / 40.0^{* \star *}$ & $14 / 42.9^{\star \star *}$ \\
\hline ALL & $593 / 30.0$ & $128 / 32.8$ & $132 / 28.0$ & $163 / 28.8$ & $170 / 30.6$ \\
\hline $\begin{array}{l}\text { F-Test } \\
\text { (Probability) }\end{array}$ & $3.93(0.009)^{\star \star \star}$ & $5.05(0.00)^{\star \star \star}$ & $3.46(0.02)^{\star \star}$ & $0.60(0.61)$ & $1.49(0.14)$ \\
\hline
\end{tabular}

* Significant at a $90 \%$ level of confidence.

** Significant at a $95 \%$ level of confidence.

$\star \star \star \star$ Significant at a $99 \%$ level of confidence. 
award percentage for the respective round; indeed it was significantly lower in round II. For TIGER II, the Probability of Net Benefits rating score of 2 was actually greater than average. For rounds IV and V, the results for the tests of equality are not statistically significant. For TIGER V, the only rating category with an award percentage that was significantly different than the average was the highest rated category of 5 , which was significantly higher. For the combined rounds and the individual rounds, both the tests of equality and the simple hypotheses tests indicate that the RT seemed to view the highest rated projects favorably but was relatively indifferent to projects with different ratings.

For the BCA-Usefulness variable, we reject the null hypothesis of equality for the combined rounds and for rounds II-III. For TIGER V, the result almost rises to an accepted level of significance $(p=0.14)$. For the combined rounds, over half of the applications where the Econ staff rated the analysis as Very Useful received an award and this was significantly higher than average. However, nearly 30\% of projects with Not Useful BCAs received an award. For TIGER II, both the top categories (Useful and Very useful) had award percentage higher than 50\% and the percentages were significantly higher than average. However, for TIGER III it is the high percentage of awarded Very Useful rated projects that drive the results. In TIGER III, the award percentage for projects with Useful BCAs was less than average, and for that matter, less than the percentage for projects with Not Useful BCAs. For rounds IV and V, the results for the tests of equality are not statistically significant. For both rounds, projects with Very Useful BCAs had a significantly higher award percentage but in both cases the award percentage for projects with Useful BCAs was below average. Indeed, for TIGER IV the award percentage for projects with Useful BCAs was significantly less. For the combined rounds and the individual rounds, both the tests of equality and the simple hypotheses tests indicate that the RT seemed to view the projects with Very Useful BCAs favorably but was relatively indifferent to projects with different Usefulness ratings.

As a whole, these results tend to confirm the results in Table 3 concerning the influence of both BCA-Usefulness and Probability of Net Benefits in TIGER IV and V. These results would seem to indicate that projects with the highest rated BCAUsefulness and Probability of Net Benefits have a positive effect in the selection process but that effect has decreased in recent rounds.

An alternative method of answering Hypothesis \#2 is to do N-Way tabulations where we compare the actual count (not percentage) of awarded and nonawarded project by rating category. The test is a Pearson Chi-square $\left(\chi^{2}\right)$ test for overall independence among all series in the group. The test statistic is a $\chi^{2}$ statistic and the null hypothesis is independence. Consequently, a statistically significant test statistic implies that there is a relationship between ratings and awards. Equation (5) shows the test equation. In the equation, $n_{i j k}$ and $N_{i j k}$ are the actual 
and overall expected counts in each cell and $i, j$, and $k$ are the number of categories for each series.

$$
\chi^{2}=\Sigma_{i j k} \frac{\left(N_{i j k}-n_{i j k}\right)^{2}}{N_{i j k}}
$$

Table 6 shows the results for the Probability of Net Benefits and Table 7 shows the results for BCA-Usefulness. For the combined rounds of TIGER, we reject independence for both Probability of Net Benefits and BCA-Usefulness. This suggests a relationship between ratings and awards and it tends to confirm the results from the test of equality (Tables 4 and 5). However, we do not have any way of knowing how much the relationship between the highest rated categories and getting an award drives the results. We do not show the results separately for each round, but for both Probability of Net Benefits and BCA-Usefulness we reject independence for TIGER II and III but we were not able to do so for rounds IV and V. These results suggest that the relationship between ratings and awards has become weaker over the latest rounds of TIGER. This also tends to confirm the results from the tests of equality. One thing that is readily apparent from both Tables 6 and 7 is the large number of projects with lower quality BCA's and lower likelihood of positive net benefits that received awards. Nearly 65\% of awarded projects had BCA's rated Not Useful or Marginally Useful. Additionally, just over

Table 6 Pearson test for Probability of Net Benefits ordinal rating scores for TIGER Rounds II-V.

\begin{tabular}{lrrrrrr}
\hline & $\mathbf{1}$ & $\mathbf{2}$ & $\mathbf{3}$ & $\mathbf{4}$ & $\mathbf{5}$ & Total \\
\hline No Award & 6 & 49 & 169 & 139 & 52 & 415 \\
Award & 0 & 16 & 65 & 55 & 42 & 178 \\
Total & 6 & 65 & 234 & 194 & 94 & 593 \\
Pearson test (probability) & $13.92(0.008)^{* * *}$ & & & & & \\
\hline
\end{tabular}

${ }^{\star * \star}$ Significant at a $99 \%$ level of confidence.

Table 7 Pearson test for BCA-Usefulness ordinal rating scores for TIGER rounds II-V.

\begin{tabular}{lrrrrr}
\hline & $\mathbf{1}$ & $\mathbf{2}$ & $\mathbf{3}$ & $\mathbf{4}$ & Total \\
\hline No Award & 90 & 185 & 119 & 21 & 415 \\
Award & 38 & 68 & 49 & 23 & 178 \\
Total & 128 & 253 & 168 & 44 & 593 \\
Pearson test (probability) & $11.62(0.009)^{* \star *}$ & & & & \\
\hline
\end{tabular}

***Significant at a $99 \%$ level of confidence. 
half of the awarded projects had Probability of Net Benefits scores $<4$ (uncertain but benefits likely to outweigh costs).

Homan et al. (2014) had earlier found the average quality of the BCA's for TIGER I was not good. As noted earlier, following TIGER I, US DOT made several efforts to help applicants prepare better BCAs. This included guidance in the NOFAs and webinars. In doing so, the hope was to that in improving the quality of the BCAs there would be less uncertainty surrounding the results. Consequently, it would be of value to see if there were any improvements in BCA quality over the following rounds of TIGER. We do so for the average scores for all BCAs reviewed by the Econ Team (awarded or not). We do so using the test for equality of means [Equation (1)]. The null hypothesis is that the average ratings are the same for all rounds.

\section{H5: Average scores are equal for all rounds}

If the average scores (BCA-Usefulness or Probability of Net Benefits) are progressively higher with each subsequent round, we'd expect that it is likelier for the differences to be significant. Conversely, if the range between the average scores is relatively flat and the individual round averages are all close to the total average, then it is less likely that the differences would be significant. However, even if most individual round averages are close to the total average we can still reject the null hypothesis if there is a significant outlier (e.g. one rating has a much higher average score than the average for all rounds). As with the ordinal ratings test, we also conduct a simple hypothesis test [Equation (4)] between the average score for each round and the total average for all rounds. If the average scores are progressively higher with each round we'd expect to see earlier rounds with a statistically significant lower than average score. We'd also expect to see later rounds with a statistically significant higher than average score.

In addition to determining if overall quality of forwarded application quality has been increasing, we also investigate whether the quality of awarded projects has been increasing. This is more of a test of whether Econ team metrics have increasingly mattered in the selection of awardees (or not), as opposed to general quality. It is also a way to investigate whether the capability (or desire) of the selection process to discriminate between good and bad projects (based on Econ team metrics) is improving.

Table 8 shows the results of the comparisons over rounds II-V. For all forwarded applications across the four rounds of TIGER, we reject the null hypothesis of equality for both Probability of Net Benefits and BCA-Usefulness. We also find that for later rounds, both Probability of Net Benefits and BCA-Usefulness average scores were significantly higher than the average. For BCA-Usefulness the average increased following each round. However, that does not necessarily 
Table 8 Comparison of Probability of Net Benefits and BCA-Usefulness rating by TIGER round.

\begin{tabular}{lrrrrr}
\hline Round & & All & & Awardees \\
\cline { 2 - 3 } \cline { 5 - 6 } & $\begin{array}{r}\text { BCA- } \\
\text { Usefulness }\end{array}$ & $\begin{array}{r}\text { Probability of } \\
\text { Net Benefits }\end{array}$ & & $\begin{array}{r}\text { BCA- } \\
\text { Usefulness }\end{array}$ & $\begin{array}{r}\text { Probability of } \\
\text { Net Benefits }\end{array}$ \\
\hline TIGER II & $2.055^{\star \star *}$ & 3.461 & & 2.333 & 3.714 \\
TIGER III & 2.197 & $3.356^{* \star *}$ & & 2.324 & $3.568^{*}$ \\
TIGER IV & $2.282^{*}$ & $3.614^{* \star *}$ & & 2.277 & 3.723 \\
TIGER V & $2.288^{* *}$ & $3.582^{* *}$ & & 2.346 & 3.731 \\
ALL & 2.221 & 3.514 & & 2.320 & 3.691 \\
F-Test/probability & $2.23(0.08)^{*}$ & $2.40(0.07)^{*}$ & $0.05(0.99)$ & $0.27(0.85)$ \\
\hline
\end{tabular}

* Significant at a $90 \%$ level of confidence.

${ }^{* \star}$ Significant at a $95 \%$ level of confidence.

$\star \star \star$ Significant at a $95 \%$ level of confidence.

mean that scores are good or that the improvement has been uniform. Starting with TIGER II, the average BC-Usefulness rating was not very good. The average was just at a Marginal Useful (see Table 2). By TIGER V, it had improved significantly. However, even at 2.29 the average was not even close to a Useful rating and was not much different than for TIGER IV. For the Probability of Net Benefits variable, the average score went down in TIGER III and also went down in TIGER V.

While the results for all forwarded applications are mixed, the results for awarded projects are straightforward. We do not reject the null hypothesis of equality for both Probability of Net Benefits and BCA-Usefulness. The range between individual rounds is very flat. Indeed, for BCA-Usefulness not any individual round average is significantly different than the total. The results for only awarded projects indicate that the emphasis on Econ team metrics like BCA-Usefulness in the award process has not been increasing.

We also conduct a test of equality between individual rounds (e.g. TIGER II to TIGER III) to determine if there has been a significant increase in scores round-toround. Table 9 shows the results. For all applications, the increase in the average BCA-Usefulness score from one round to the next was not significant for any of the round-to-round changes. The closest was the change from TIGER II to III, but it did not quite rise to an accepted level of significance $(\mathrm{p}=0.17)$. However, the direct comparison between round II and V is significant. The overall increase is consistent with the F-Test results from Table 8. For Probability of Net Benefits, there was only a significant increase from II to III. This lone round-to-round result is likely driving the significance level in the F-Test in Table 8. For awarded projects only, there were not any significant increases from one round to the next for either variable. 
Table 9 Comparison of round-to-round changes in Probability of Net Benefits and BCA-Usefulness.

\begin{tabular}{|c|c|c|c|c|}
\hline \multirow[t]{3}{*}{ Rounds } & \multicolumn{2}{|r|}{ All } & \multicolumn{2}{|r|}{ Awardees } \\
\hline & BCA-Usefulness & $\begin{array}{r}\text { Probability of Net } \\
\text { Benefits }\end{array}$ & BCA-Usefulness & $\begin{array}{r}\text { Probability of Net } \\
\text { Benefits }\end{array}$ \\
\hline & F-Test/probability & F-Test/probability & F-Test/probability & F-Test/probability \\
\hline TIGER II to III & $1.87(0.17)$ & $1.05(0.31)$ & $0.00(0.97)$ & $0.45(0.50)$ \\
\hline TIGER III to IV & $0.72(0.40)$ & $5.83(0.02)^{* *}$ & $0.05(0.82)$ & $0.66(0.42)$ \\
\hline TIGER IV to $\mathrm{V}$ & $0.00(0.95)$ & $0.08(0.77)$ & $0.14(0.71)$ & $0.00(0.97)$ \\
\hline TIGER II to V & $5.27(0.02)^{\star \star}$ & $1.26(0.26)$ & $0.00(0.95)$ & $0.01(0.94)$ \\
\hline
\end{tabular}

**Significant at a $95 \%$ level of confidence.

For BCA-Usefulness in particular, the small, but overall rise in the BCA quality for all applicants coupled with not any change in BCA quality for awarded projects since TIGER II explains its declining influence in the selection process seen in Table 3. This also reflects the reduced percentage of higher rated projects (both Probability of Net Benefits and BCA-Usefulness) receiving awards in TIGER IV and TIGER V (Tables 4 and 5).

\section{Conclusion}

This paper presents a review of the role of benefit-cost analysis (BCA) in the TIGER grant review process. In particular, it discusses many of the common errors found in the BCAs prepared by applicants. Because of these errors it was not possible to precisely quantify benefits for most projects or to arrive at a benefit-cost ratio (BCR) for the projects. Because of staff and time constraints, it was not possible to independently conduct internal BCAs for all projects to arrive at a BCR.

However, it was important to still be able to provide information on both the quality of the analysis and more importantly the likely net benefits of each project to the senior review team (RT) of high level DOT officials that drove the selection process. The paper discusses metrics DOT staff developed to do so. The metrics also allow us to analyze whether the quality of the BCA and the likelihood that benefits exceeded costs (net social benefits) influenced whether or not DOT selected a project for funding.

A secondary focus of the paper was to then analyze whether the usefulness of the BCA and the likelihood that the benefits exceeded the costs (both, as identified by DOT) influenced the selection process. To do so, we analyze eligible 
projects reviewed by the RT where DOT staff had also reviewed the BCA. We do so for TIGER rounds II-V. We found that for earlier rounds of TIGER, the likelihood of positive net benefits and BCA quality was higher for TIGER grant awardees than non-awardees and that the differences were statistically significant. This would indicate that the likelihood of net societal benefits mattered to the RT. However, the influence of these variables has diminished over later rounds of TIGER. For all rounds, there was a large number of projects with lower quality BCAs that received awards, as well as a relatively high percentage of projects with lower quality BCAs that received awards.

Acknowledgments: Many thanks to Jack Wells for useful inputs throughout the process and to an anonymous referee for helpful comments. I would also like to thank Ryan Endorf for assistance in conducting the research.

\section{References}

Boardman, A., Greenberg, D., Vining, A., \& Weimer, D. (2006). Cost-benefit analysis concepts and practice. Upper Saddle Ridge, NJ: Pearson Prentice Hall.

Flyvbjerg, B., Holm, M., \& Buhl, S. (2005). How (in)accurate are demand forecasts in public works projects? the case of transportation. Journal of the American Planning Association, 71(2), 131-146.

Government Accountability Office (GAO). (2011a). Competitive grant programs could benefit from increased performance focus and better documentation of key decisions (GAO-11-234). Retrieved from http://www.gao.gov/assets/320/317196.pdf.

Government Accountability Office (GAO). (2011b). High-risk series: an update (GAO-11-278). Retrieved from http://www.gao.gov/assets/320/315725.pdf.

Homan, A., Adams, T., \& Marach, A. (2014). A statistical analysis of the role of benefit-cost analysis in awarding TIGER grants. Public Works Management and Policy, 19(1), 37-50.

Notice of Funding Availability for Supplemental Discretionary Grants for Capital Investments in Surface Transportation Infrastructure Under the American Recovery and Reinvestment Act (NOFA 2009). (2009). Federal Register, 74(94), 23226-23237.

Notice of Funding Availability for the Department of Transportation's National Infrastructure Investments Under the Transportation, Housing and Urban Development, and Related Agencies Appropriations Act for 2010 (NOFA 2010). (2010). Federal Register, 75 , 30460-30480.

Notice of Funding Availability for the Department of Transportation's National Infrastructure Investments Under the Full-Year Continuing Appropriations 2011 (NOFA 2011). (2011). Federal Register, 76, 50289-50312.

Notice of Funding Availability for the Department of Transportation's National Infrastructure Investments under the Full-Year Continuing Appropriations 2012 (NOFA 2012). (2012). Federal Register, 77(20), 4863-4880. 
Notice of Funding Availability for the Department of Transportation's National Infrastructure Investments Under the Consolidated and Further Continuing Appropriations Act, 2013 (NOFA 2013). (2013). Federal Register, 78, 24786-24794.

Office of Management and Budget (OMB). (2003). Circular A-4. Retrieved from http://www. whitehouse.gov/omb/circulars_a004_a-4.

Quantitative Micro Software. (2007). EVIEWS User's Guide I. Irvine, CA: Quantitative Micro Software.

Article note: The US Department of Transportation (DOT) disclaims responsibility for any private publication or statement by any of its employees. The views expressed herein are those of the author and do not necessarily reflect the views of the DOT. 